\title{
EVALUACIÓN POBLACIONAL DEL VENADO COLA BLANCA EN UN BOSQUE TROPICAL SECO DE LA MIXTECA POBLANA
}

\author{
M. Concepción LóPEZ-TÉLLEZ1, \\ Salvador MANDUJANO ${ }^{2}$ \& Gonzalo YÁNES ${ }^{1}$ \\ ${ }^{1}$ Escuela de Biología, Benemérita Universidad Autónoma de Puebla, Puebla, MÉXICO. \\ cs001414@siu.buap.mx \\ 2 Departamento de Biodiversidad y Ecología Animal, Instituto de Ecología A. C., km 2.5, \\ Carretera Antigua a Coatepec No. 351, Congregación del Haya, Xalapa 91070, \\ Veracruz, MÉXICO. salvador.mandujano@inecol.edu.mx
}

RESUMEN

Con el objetivo de diagnosticar el potencial de aprovechamiento y prioridades de manejo en UMAs extensivas, se estimó la densidad poblacional del venado cola blanca (Odocoileus virginianus mexicanus) y se caracterizó el hábitat de bosque tropical seco en cuatro localidades de la Mixteca Poblana, México. En cada localidad se establecieron cinco transectos para el conteo de grupos fecales de marzo del 2000 a marzo de 2001. Se aplicó un análisis de componentes principales para describir el hábitat y su asociación con la densidad de venados. La densidad varió notablemente entre localidades, transectos y meses de muestreo. La densidad promedio fue de $1.8 \pm 0.01$ venados $/ \mathrm{km}^{2}$. Las localidades de Mitepec y El Salado tuvieron densidades más altas (3.2 a 3.4 venados $/ \mathrm{km}^{2}$ ), mientras que en Huachinantla y Jolalpan fueron más bajas $\left(0.1\right.$ a 0.5 venados $\left./ \mathrm{km}^{2}\right)$. La densidad estuvo asociada con la altitud, cobertura térmica y de protección del sotobosque. Estas condiciones varían entre las localidades lo que podría explicar las diferencias en la densidad de venados. Desde una perspectiva de aprovechamiento, nuestros resultados sugieren las siguientes acciones: veda para recuperar las poblaciones de venados, planeación del manejo del hábitat, y programas de participación comunitaria para involucrar a los pobladores de las localidades en el manejo sustentable del venado.

Palabras Clave: Bosque tropical seco, densidad, Mixteca, Puebla, venado cola blanca.

\begin{abstract}
To evaluate the potential use and management in extensive UMAs, we estimated the population density of the white-tailed deer (Odocoileus virginianus mexicanus) and the habitat characteristics in a tropical dry forest from four towns of the Mixteca Poblana, Mexico. In each location, five transects were established to count the fecal groups from March 2000 to March 2001. Principal components analysis was applied to describe the habitat and the association with deer density. The density varied notably among locations, transects and months of sampling. The average density was $1.8 \pm 0.01 \mathrm{deer} / \mathrm{km}^{2}$. The locations of Mitepec and El Salado had higher densities (3.2 to 3.4 deer $/ \mathrm{km}^{2}$ ), while in Huachinantla and Jolalpan they were lower $\left(0.1\right.$ to $\left.0.5 \mathrm{deer} / \mathrm{km}^{2}\right)$. The density was associated with elevation, thermal cover, and protection cover. These conditions vary among the locations, which could explain the
\end{abstract}


differences in the population density. From human use perspective, our results suggest actions be taken to regulate the hunting, to recover the habitat, and include the participation of local people for a sustainable management of the deer.

Key Words: Tropical dry forest, population density, Puebla, Mixteca, white-tailed deer.

\section{INTRODUCCIÓN}

Por su gran adaptabilidad, tamaño corporal, amplia distribución geográfica, la aceptación de su carne para consumo humano y como trofeo cinegético, el venado cola blanca (Odocoileus virginianus) es una de las especies más intensamente manejadas en criaderos y vida silvestre en México (Galindo-Leal y Weber 1998; Villarreal 1999). Este interés por el venado se ha extendido ampliamente en el Estado de Puebla donde la subespecie O. v. mexicanus está siendo manejada en UMAs intensivas y extensivas (Romero y Martínez-Romero 1998; Villarreal-Espino 1998, 2000, 2002; Romero 1999; Romero y Villarreal-Espino 2000; Villarreal-Espino et al. 2000; Delfín 2002; Durán et al. 2004; Martínez-Romero 2004; López-Benítez et al. 2004; Pérez-Mejía et al. 2004; Villarreal-Espino y Marín 2005; Villarreal-Espino et al. 2005). En particular, la Mixteca Poblana es una de las regiones más extensas del Estado, dominada por bosque tropical seco, y con un alto grado de perturbación y marginación (Gobierno del Estado de Puebla 1993). Dentro de las actividades que se desarrollan se tiene a la agricultura de temporal, la minería, la ganadería y la silvicultura, las cuales son económicamente improductivas y al practicarse se provoca la degradación de los suelos, y por lo tanto, de sus recursos naturales.

Para resolver la problemática en la Mixteca Poblana es necesario elaborar alternativas de desarrollo socioeconómico que permita a sus habitantes mejorar la calidad de vida incrementando la producción sin deteriorar el medio ambiente. Una alternativa es el aprovechamiento sustentable del venado cola blanca de la subespecie mexicanus (Villarreal-Espino y Guevara 2002). En este sentido, en México recientemente se ha establecido el Grand-slam de venado cola blanca el cual incluyó inicialmente solo a cuatro subespecies norteñas (O.v. texanus, O.v. couesi, O.v. carminis y O.v. miquihuanensis), pero recientemente se ha incluido a O.v. mexicanus en dicho evento (Villarreal-Espino 2002). En la actualidad en ocho municipios de Mixteca poblana se encuentran operando un total de once UMAs (Unidades de Conservación, Manejo y Aprovechamiento Sustentable) de venado cola blanca mexicano, que abarcan un total de 14,334 ha siendo el potencial de la región de más de 500,000 ha (Villarreal-Espino 2000). El presente estudio se encuentra dentro de este problemática y alternativa productiva del venado cola blanca mexicano en UMAs extensivas de la Mixteca. Los objetivos de este trabajo fueron estimar la densidad poblacional del venado cola blanca, caracterizar el bosque tropical seco, y conocer la asociación entre la densidad y algunas variables del 
hábitat, en cuatro localidades del Municipio de Jolalpan. Esto se hizo con la finalidad de tener un primer diagnóstico de la población de venados y proponer áreas prioritarias para el aprovechamiento y la conservación de esta especie en la región de la Mixteca Poblana.

\section{MÉTODOS}

\section{1. Área de estudio}

El trabajo se llevó a cabo en el Municipio de Jolalpan que se localiza en la parte suroeste del Estado de Puebla (Fig. 1; $18^{\circ} 12^{\prime} 18^{\prime \prime} \mathrm{N}$ y $98^{\circ} 46^{\prime} 24^{\prime \prime} \mathrm{O}$ ). Tiene una superficie de $424 \mathrm{~km}^{2}$ y para el año 2000 contaba con una población humana de 12,556 habitantes (Centro Nacional de Estudios Municipales 2000). Las principales actividades económicas que se practican son la agricultura de temporal, la fruticultura y de hortalizas, además de la ganadería. El Municipio se encuentra dentro de la subcuenca del río Nexapa afluente del Atoyac, que a su vez es tributario del Balsas (INEGI 1996). El clima es de tipo cálido subhúmedo con lluvias en verano; la temperatura media anual es de $25.6^{\circ} \mathrm{C}$, con el mes más caliente en mayo $\left(29.1^{\circ} \mathrm{C}\right)$ y el más frío en enero $\left(22.4^{\circ} \mathrm{C}\right)$. La precipitación total anual es de $852 \mathrm{~mm}$; el mes más lluvioso es junio, mientras que febrero es el mes más seco. El tipo de vegetación que predomina es el bosque tropical seco situado entre los 1,000 y 1,800 msnm; los árboles tienen una altura que oscila entre los 8 y $12 \mathrm{~m}$ pero algunos alcanzan hasta los $30 \mathrm{~m}$ de altura. La estacionalidad es muy marcada por lo que la pérdida de hojas
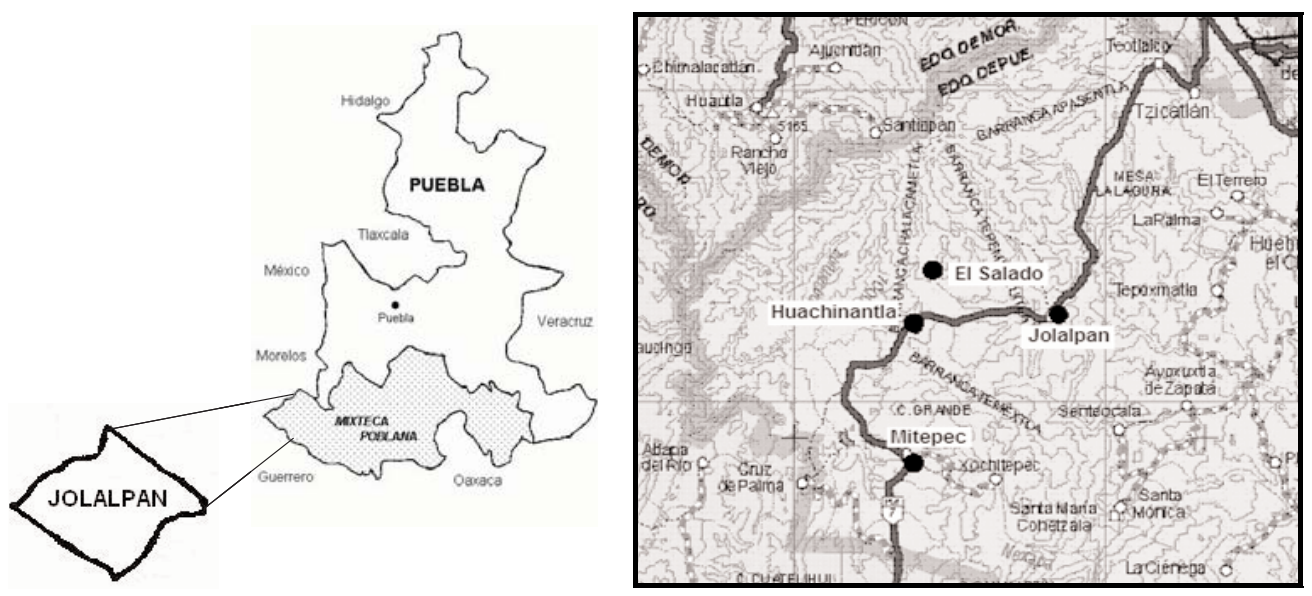

Figura 1

Localización geográfica del municipio de Jolalpan en la Mixteca Poblana, y ubicación de las cuatro localidades de estudio en El Salado, Huachinantla, Jolalpan y Mitepec. 
ocurre durante un período de 5 a 8 meses (Rzedowski 1978). Algunas de las especies más comunes son el tepehuaje (Leucaena pueblana), amapola (Pseudobombax palmeri), colorín (Eritrina spp. ), pochote (Ceiba parvifolia) palo blanco (Conzattia multiflora), cuajilote (Acacia acatlensisi), copal (Bursera bipinnata, Bursera excelsa), cueramo (Cordia spp.), cuachalalate (Amphiterygium adstringens), guaje (Leucaena glauca) huizache (Acacia farenesiana) cubata (Acacia cymbispina).

\section{Estimación de la densidad de venados}

Se trabajó en cuatro localidades: Huachinantla, Mitepec, El Salado y La Cabecera Municipal de Jolalpan (de aquí en adelante referido solo como Jolalpan), las cuales suman un área de 45,249 ha (Fig. 2). En cada localidad se establecieron cinco transectos con una distancia mínima entre ellos de un kilómetro. Se trató de que cada transecto fuera lo más recto posible, sin embargo en varias ocasiones no se logró hacer esto debido a lo accidentado del terreno. Cada transecto tuvo un largo de $400 \mathrm{~m}$ con 40 parcelas circulares de $9.3 \mathrm{~m}^{2}$ ubicadas a intervalos de $10 \mathrm{~m}$. Este tamaño de parcela representa el compromiso óptimo entre la varianza de muestreo la cual disminuye conforme aumenta el tamaño de la parcela, y el sesgo del muestreo el cual aumenta conforme es mayor el tamaño de la parcela (Smith 1968). Las parcelas se limpiaron de excrementos viejos en marzo de 2000, y luego se hicieron siete muestreos hasta marzo del 2001. Siguiendo el criterio empleado por Gallina (1990), se consideró como un grupo fecal a partir de encontrar de cinco o más bolitas con las mismas características.

Para determinar la densidad poblacional se empleó el modelo de Eberhardt y Van Etten (1956). La fórmula para convertir número de grupos fecales a número de venados por kilómetro cuadrado es:

$$
D=\frac{107,526 \times P G}{T P \times T D}
$$

donde 107,526 es el número de parcelas de $9.3 \mathrm{~m}^{2}$ en un $\mathrm{km}^{2}$, PG es el promedio de grupos de excrementos por parcela, TP es el número de días de depósito de los excrementos y TD es la tasa de defecación (grupos fecales/ individuo/ día). La densidad se obtuvo mensualmente para cada localidad. El error estándar se estimó aplicando el modelo de la binomial negativa como:

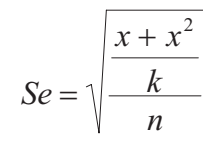

donde $\mathrm{x}$ es el promedio de grupos fecales por parcelas, $\mathrm{K}$ es el coeficiente de la binomial negativa y n el número de parcelas (Ezcurra y Gallina 1981). Para conocer si la densidad varía entre localidades, mes de muestreo o la interacción de ambos, se aplicó una análisis de varianza de dos vías considerando a los transectos de cada localidad como réplicas. 

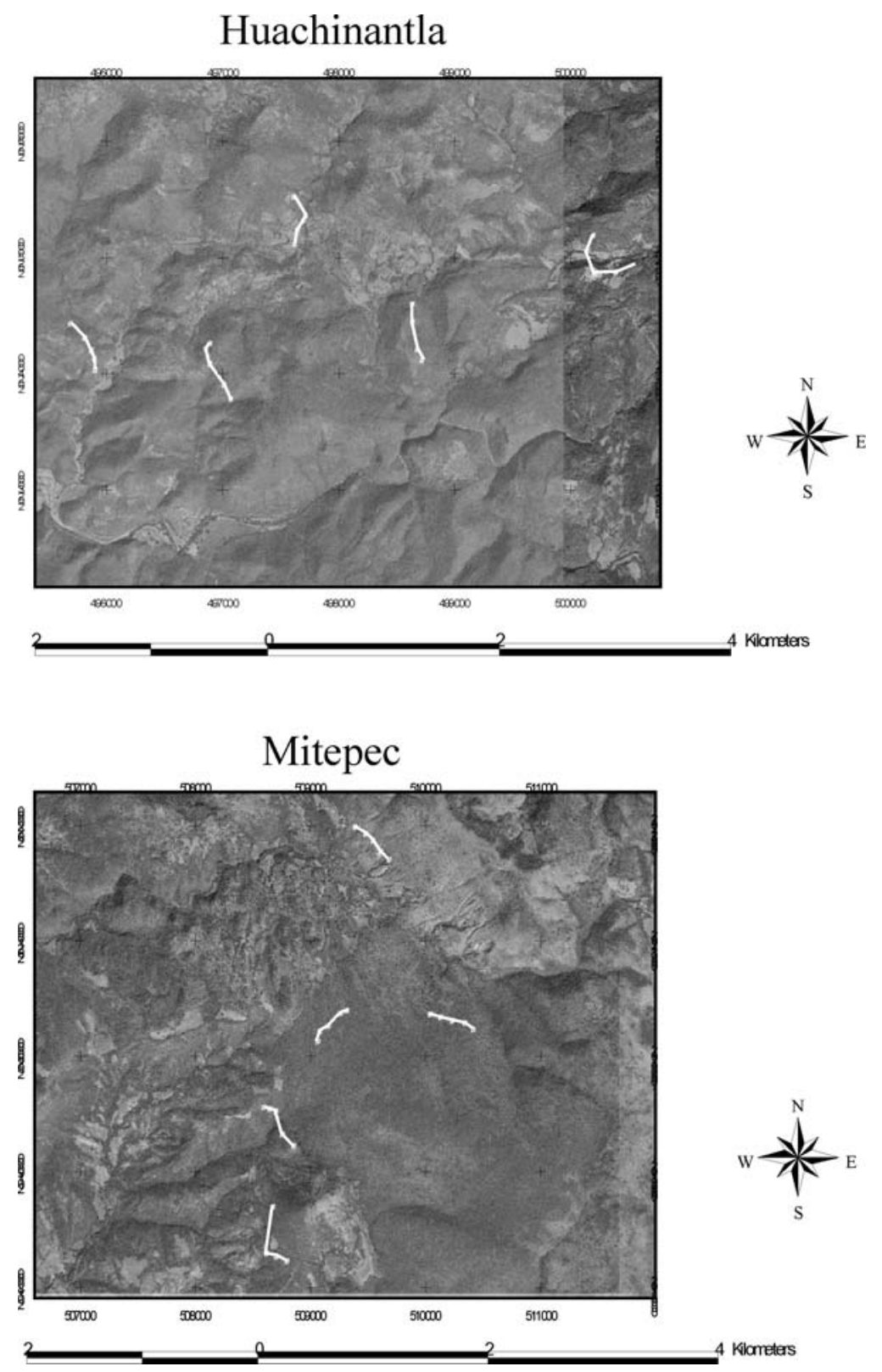

Figura 2

Ubicación de los transectos de muestreo en cada localidad de trabajo. 

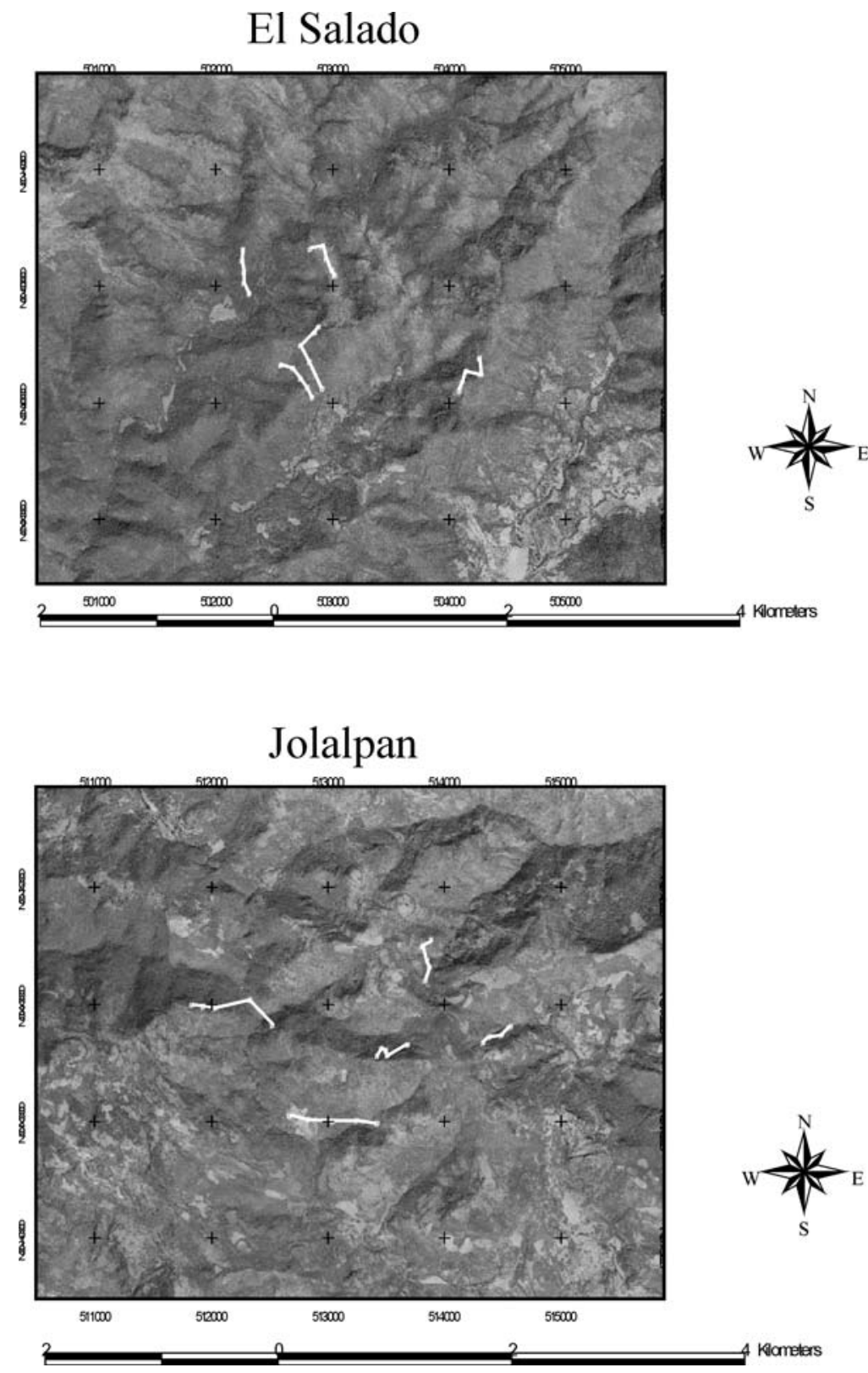
Recientemente se calculó la tasa de defecación para esta subespecie con venados en cautiverio en el Parque Estatal Lázaro Cárdenas Flor del Bosque en el Estado de Puebla (Pérez-Mejía et al. 2004). Estos autores reportan un promedio de 17 grupos/individuo/día con un rango de 8 a 25. Si bien esas estimaciones no están exentas de posibles sesgos debido al empleo de animales en cautiverio, por el momento es la única disponible al sitio de estudio y se decidió emplearla con precaución. En el modelo de Eberhardt y Van Etten (1956) la estimación de la densidad está influenciada de manera importante por la tasa de defecación, pues esto permite convertir a número de animales por unidad de área. Puesto que en el área de estudio se pretende hacer aprovechamiento de los venados a partir de cacería controlada, en este estudio decidimos emplear la tasa de 25 grupos/individuo/día que representa el valor máximo obtenido con los animales en cautiverio. Además, hay datos que sugieren que la tasa de defecación de venados en vida libre es más alta en comparación a venados en cautiverio, debido a la variación en la cantidad de fibra y humedad de las plantas consumidas (Neff 1968; Rogers 1987). En consecuencia emplear la tasa de 25 no sobreestimaría la densidad de venados en las localidades de estudio, lo cual permite de este modo proteger a la población pues las tasa de aprovechamiento se basaría en una estimación conservadora de la población. Sin embargo, reconocemos que a largo plazo emplear esta tasa de defecación podría llevar a un sub-aprovechamiento de las poblaciones de venado por lo que, en su momento, habrá que reconsiderar el empleo de dicha tasa.

\section{Caracterización del hábitat y asociación con la densidad de venados}

Para caracterizar el hábitat se hicieron muestreos cada $20 \mathrm{~m}$ en los mismos transectos empleados para el conteo de grupos fecales. La caracterización incluyó variables vegetales (densidad, altura, área basal y cobertura de copa del estrato arbóreo, y cobertura de protección de 0 a 50, 51 a 100, 101 a 150 y 151 a $200 \mathrm{~cm}$ del nivel de suelo) y variables físicas (altitud, pendiente, orientación de ladera, sinuosidad, y distancia de los transectos al cuerpo de agua más cercano). Para la evaluación del estrato arbóreo en cada transecto se aplicó el método de cuadrante centrado en puntos (Muller-Dombois \& Ellenberger 1974); mientras que la cobertura de protección se estimó siguiendo el procedimiento descrito por Griffith y Youtie (1988). Se vació toda la información a una base de datos y se generaron mapas con los programas Arc view 3.2a y Golden Surfer 7.0 con auxilio de la carta digitalizada de la zona y ortofotos (INEGI 1987, 1996, 1997, 1998).

Para ordenar los transectos en función de las características del hábitat, se realizó un análisis de componentes principales considerando una matriz de 13 variables de hábitat y 20 transectos de muestreo. Para estandarizar los datos se empleó la matriz de correlación. Para estimar la contribución relativa de cada variable en los primeros tres componentes principales y el nivel de significancia de cada una, se empleó el 
programa Statistica 4.3. A partir de estos resultados se graficaron los primeros dos componentes para mostrar la ordenación de los transectos en función de las variables que significativamente contribuyen en los componentes. Se trazó un polígono uniendo los transectos de cada localidad con el fin de visualizar el grado de heterogeneidad de cada localidad.

\section{RESULTADOS}

\section{Densidad poblacional}

Se contaron un total de 94 grupos fecales en los 800 parcelas de los 20 transectos visitados durante siete muestreos en las cuatro localidades de estudio. En general, considerando las cuatro localidades de estudio de manera simultánea, la densidad poblacional promedio fue de $1.8 \pm 0.01$ venados $/ \mathrm{km}^{2}$. En particular, las estimaciones fueron significativamente más altas en El Salado y Mitepec con 3.4 y 3.2 venados $/ \mathrm{km}^{2}$, respectivamente; mientras que Jolalpan y Huachinantla se estimaron solo 0.5 y 0.1 venados $/ \mathrm{km}^{2}$ (Cuadro $1 ; \mathrm{F}=3.62, \mathrm{gl}=3,112 \mathrm{P}=0.02$ ).

Las estimaciones de la densidad variaron notablemente dependiendo de la localidad, transecto y mes de muestreo (Cuadro $1 ; \mathrm{F}=2.43, \mathrm{gl}=18,112 \mathrm{P}=0.002$ ). Tanto en El Salado y Mitepec hubo presencia de venados en todos los transectos; mientras que en Huachinantla y Jolalpan solo se detectó presencia en uno o dos transectos. Por otro lado, no hubo un solo transecto en el cual se registrara la presencia de venados durante los siete meses de muestreo (Cuadro 1). Incluso en El Salado y Mitepec hubo transectos en los que solo una vez durante los siete meses se detectaron grupos fecales. El extremo es Huachinantla donde solo en dos transectos en un mes se detectó presencia de venados. En cada localidad las estimaciones de la densidad variaron notablemente de un mes a otro (Cuadro 1). En algunos casos esta variación fue extrema, por ejemplo en El Salado durante el mes de enero se estimó hasta 17.8 venados $/ \mathrm{km}^{2}$, mientras que en el mes previo y el posterior se estimó solo 0.7 venados $/ \mathrm{km}^{2}$.

\section{Caracterización del hábitat}

Las características físicas y vegetales del hábitat variaron entre localidades, y entre transectos dentro de una misma comunidad (Cuadro 2). Al resumir esta variación en el análisis de componentes principales, se encontró que los tres primeros componentes explicaron el $67 \%$ de la variación de la matriz. Correlacionados significativamente con el primer componente se tuvieron a las variables altitud, cobertura térmica y cobertura de protección de 101 a $150 \mathrm{~cm}$ del suelo; mientras que en el segundo componente se tuvo a la cobertura de protección de 0 a $50 \mathrm{~cm}$ del suelo. 


\section{Cuadro 1}

Estimaciones mensuales, por transecto y promedio de la densidad poblacional (individuos $/ \mathrm{km}^{2}$ ) del venado cola blanca en cada localidad de estudio.

\begin{tabular}{llc|c}
\hline \multirow{3}{*}{ Localidad/transecto } & \multicolumn{2}{c}{2001} \\
\cline { 2 - 4 } Jun Jul Ago-Sep Oct-Nov Dic & Ene Mar \\
\hline
\end{tabular}

El Salado

$\begin{array}{ccccccccccc} & \text { T1 } & 0.0 & 0.0 & 0.0 & 0.0 & 0.0 & 7.4 & 0.0 & 1.1 & 0.01 \\ & \text { T2 } & 0.0 & 0.0 & 0.0 & 0.0 & 0.0 & 3.7 & 1.8 & 0.8 & 0.01 \\ & \text { T3 } & 2.2 & 0.0 & 0.0 & 0.0 & 1.7 & 18.6 & 0.0 & 3.2 & 0.02 \\ & \text { T4 } & 11.2 & 0.0 & 0.0 & 6.1 & 1.7 & 52.0 & 1.8 & 10.4 & 0.06 \\ \text { Promedio } & \text { T5 } & 4.5 & 0.0 & 0.0 & 0.0 & 0.0 & 7.4 & 0.0 & 1.7 & 0.01 \\ & & 3.6 & 0.0 & 0.0 & 1.2 & 0.7 & 17.8 & 0.7 & 3.4 & 0.02\end{array}$

Mitepec

Jolalpan

$\begin{array}{cccccccccc}\text { T1 } & 4.3 & 0.0 & 0.0 & 0.0 & 0.0 & 0.0 & 0.0 & 0.6 & 0.01 \\ \text { T2 } & 10.8 & 3.5 & 0.0 & 0.0 & 0.0 & 0.0 & 0.0 & 2.0 & 0.02 \\ \text { T3 } & 12.9 & 41.7 & 0.0 & 5.4 & 0.0 & 5.7 & 0.0 & 9.4 & 0.05 \\ \text { T4 } & 6.5 & 0.0 & 0.0 & 0.0 & 0.0 & 14.2 & 0.0 & 2.9 & 0.02 \\ \text { T5 } & 8.6 & 0.0 & 0.0 & 0.0 & 0.0 & 0.0 & 0.0 & 1.2 & 0.01 \\ & 8.6 & 9.0 & 0.0 & 1.1 & 0.0 & 4.0 & 0.0 & 3.2 & 0.02\end{array}$

$\begin{array}{cccccccccc}\text { T1 } & 0.0 & 0.0 & 0.0 & 0.0 & 0.0 & 0.0 & 0.0 & 0.0 & 0.0 \\ \text { T2 } & 0.0 & 0.0 & 0.0 & 0.0 & 0.0 & 0.0 & 0.0 & 0.0 & 0.0 \\ \text { T3 } & 0.0 & 0.0 & 0.0 & 5.5 & 6.5 & 0.0 & 5.6 & 2.5 & 0.03 \\ \text { T4 } & 0.0 & 0.0 & 0.0 & 0.0 & 0.0 & 0.0 & 0.0 & 0.0 & 0.0 \\ \text { T5 } & 0.0 & 0.0 & 0.0 & 0.0 & 0.0 & 0.0 & 0.0 & 0.0 & 0.0 \\ & 0.0 & 0.0 & 0.0 & 1.1 & 1.3 & 0.0 & 1.1 & 0.5 & 0.01\end{array}$

Promedio

Huachinantla

\begin{tabular}{lccccccccccc} 
& T1 & 0.0 & 0.0 & 0.0 & 0.0 & 0.0 & 0.0 & 0.0 & 0.0 & 0.0 \\
& T2 & 0.0 & 0.0 & 0.0 & 0.0 & 0.0 & 0.0 & 0.0 & 0.0 & 0.0 \\
& T3 & 0.0 & 0.0 & 0.0 & 0.0 & 0.0 & 0.0 & 0.0 & 0.0 & 0.0 \\
Promedio & T4 & 0.0 & 0.0 & 1.7 & 0.0 & 0.0 & 0.0 & 0.0 & 0.3 & 0.01 \\
\hline
\end{tabular}


López-Téllez et al.: Venado cola blanca de la Mixteca Poblana

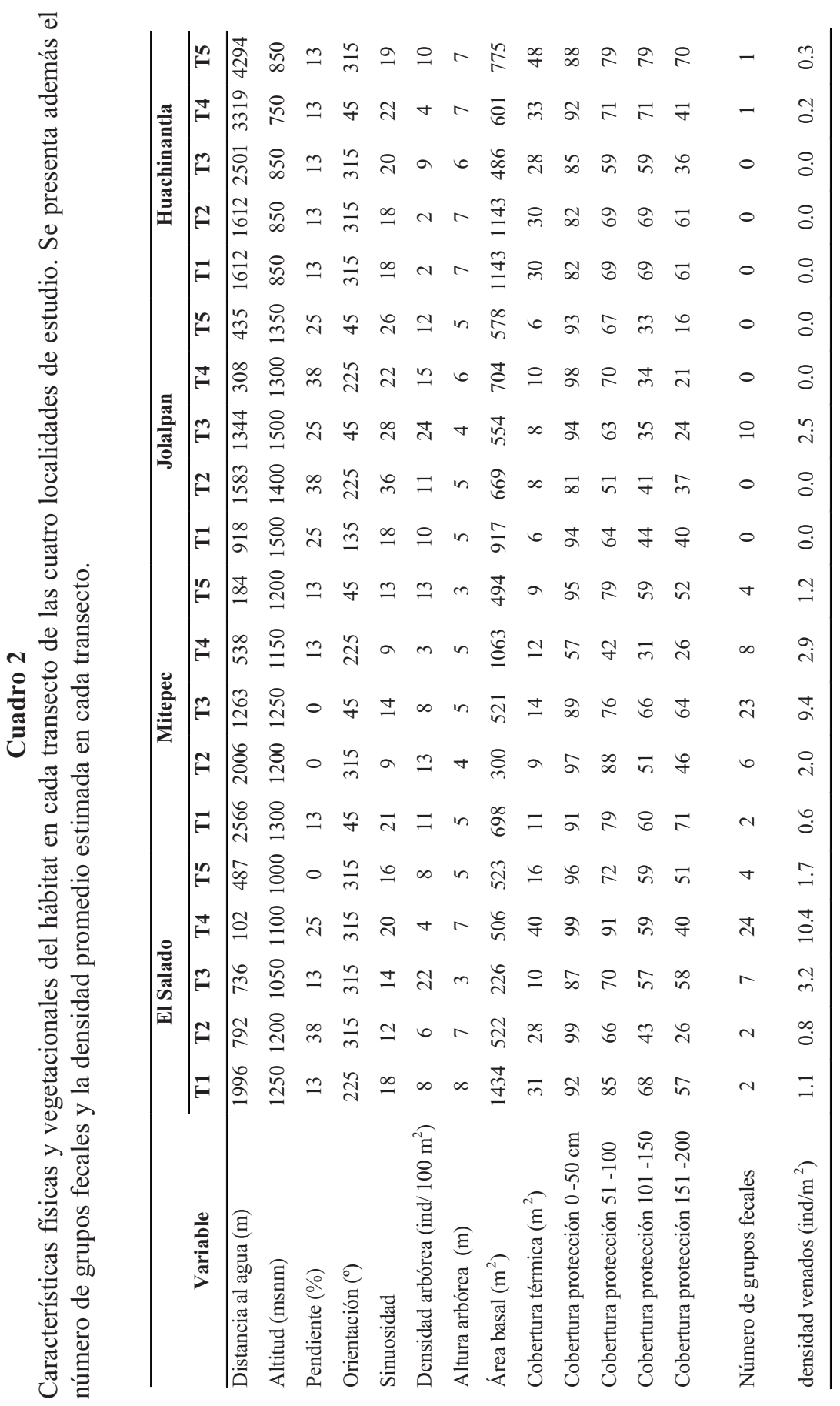


Al ordenar los 20 transectos en función de los dos componentes principales, se tuvo una clara ordenación de las cuatro localidades de estudio (Fig. 3). Por ejemplo, Jolalpan presentó la menor variación en las características del hábitat, tiene mayor altitud y coberturas térmica y de protección a 101-150 cm; mientras que Huachinantla estuvo en el extremo contrario. Mitepec presenta características del hábitat intermedias entre estas dos localidades. Mientras que El Salado es la localidad más heterogénea respecto a las variables de hábitat analizada y se traslapa bastante con Mitepec y un poco con Huachinantla (Fig. 3). Por otro lado, tanto Mitepec como El Salado tuvieron mayor cobertura del sotobosque a una altura entre 0 y $50 \mathrm{~cm}$ del suelo; mientras que Jolalpan y Huachinantla presenta menor cobertura (explicada en el componente principal dos). Es notorio que El Salado y Mitepec siendo tan similares en las condiciones de hábitat, también tuvieron similar y mayor densidad de venados, en comparación a Jolalpan y Huachinantla.

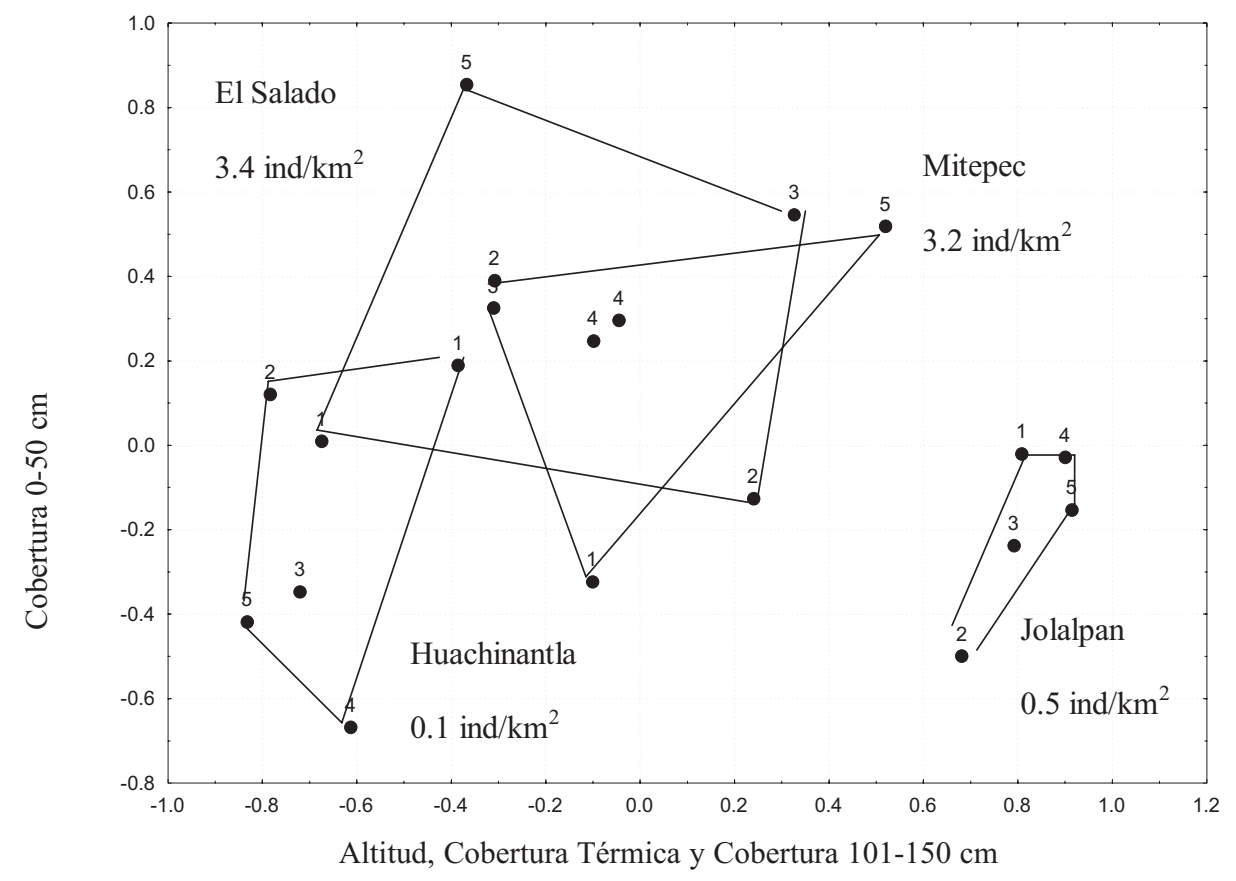

Figura 3

Ordenación de los 20 transectos de muestreo en función de las variables que se correlacionaron significativamente con el primer (altitud, cobertura térmica y cobertura de protección entre 101 y 150 $\mathrm{cm}$ del suelo) y segundo (cobertura de protección entre 0 y $50 \mathrm{~cm}$ del suelo) componente principal. En la figura se unen con un polígono los transectos de cada localidad, además se muestra la estimación de la densidad poblacional de venados. 


\section{DISCUSIÓN}

La densidad promedio del venado cola blanca estimada para en el Municipio de Jolalpan fue baja. Para poder comparar los resultados de este estudio con los obtenidos en otras áreas donde también se ha usado este método pero distinta tasa de defecación, es necesario estandarizar las estimaciones empleando la misma tasa de defecación. Por ejemplo, si consideramos la tasa de 12.7 grupos fecales por individuo al día, entonces en el área de estudio se tendría una estimación de 3.6 venados $/ \mathrm{km}^{2}$. Esta densidad es baja al compararla con la reportada en áreas protegidas de Jalisco y Durango donde se ha estimado de 14.5 a 28.1 venados $/ \mathrm{km}^{2}$ (Gallina 1990, Zavala 1992, Mandujano y Gallina 1995). La densidad en Jolalpan es similar a la reportada por Valenzuela (1991) de 4.8 venados $/ \mathrm{km}^{2}$ en un bosque templado de Jalisco; y mayor a la reportada en un bosque tropical de Colima y templado en Oaxaca de 1.8 y 1.5 venados $/ \mathrm{km}^{2}$, respectivamente (Castillo 1998, Ortiz et al. 2005). Diferencias en las características del hábitat y en la historia de uso del venado en cada localidad, podrían explicar estas diferencias. Al mismo tiempo, diferencias en la capacidad de carga debe influir en este aspecto sin embargo no sabemos cual es el potencial del hábitat en el sitio de estudio en la Mixteca Poblana como para evaluar si la densidad está o no por debajo de la capacidad de carga.

La estimación de la densidad está influenciada, entre otros factores, por la tasa de defecación empleada. La tasa de defecación puede variar notablemente dependiendo de la cantidad de fibra y humedad que ingieran los animales lo cual está relacionado a la época del año, la edad y sexo de los animales, el nivel de estrés, entre otro factores (Wallmo et al. 1962, Van Etten y Bennett 1965, Neff 1968, Wigley y Jonson 1981, Freddy y Bowden 1983, Rogers 1987, Dietrich et al. 1990, Sawyer et al. 1990). En la mayoría de los estudios donde se ha empleado el método de conteo de grupos fecales para estimar la densidad, se ha utilizado la tasa de defecación de 12.7 grupos fecales/individuo/día (e.g., Gallina 1990, Valenzuela 1991, Castillo 1998, Ortiz et al. 2004), aunque existen trabajos en los que se han empleado otras tasas (e.g. Zavala 1992, Mandujano y Gallina 1995). Por tal motivo, en el presente estudio se utilizó la tasa de defecación reportada para el venado cola blanca de la subespecie mexicanus que se encuentra en cautiverio en el Estado de Puebla (Pérez-Mejía et al. 2004). Aunque dicha estimación de la tasa de defecación no está ausente de sesgos, se seleccionó debido a que es preferible subestimar la densidad poblacional para protegerla de una sobrecosecha.

Las estimaciones de la densidad variaron notablemente entre localidades, y entre transectos en una misma localidad dependiendo del mes de muestreo. Esta variación puede deberse tanto al componente espacial como al temporal en la fluctuación de la densidad debido a cambios en el uso del hábitat por parte de los individuos y posiblemente cambios demográficos debido a la natalidad y mortalidad. Nuestros datos no permiten discernir cual de estos dos factores afecta en mayor grado la estimación de la variación de la densidad. Sin embargo, observando los cambios tan 
notorios de la densidad de un mes a otro en la misma localidad (ver Cuadro 1), sospechamos que el cambio en el uso del hábitat por los individuos puede afectar más las estimaciones de la densidad. En otros estudios también se reportan cambios notorios en la densidad de un mes a otro (e.g., Ortiz-Martínez et al. 2005). Estos cambios bruscos no pueden explicarse por variaciones en las tasas de natalidad y de mortalidad. Si la densidad de venados es baja, los individuos se mueven constantemente en un área de acción relativamente grande, el tamaño de las parcelas es pequeño, y es bajo el número de parcelas de muestreo, entonces la probabilidad de que dejen excremento en las parcelas de muestreo es baja y esto se refleja en una alta variabilidad en los datos y en las subsecuentes estimaciones de la densidad. Esto sugiere la necesidad de analizar incluso experimentalmente este método con el fin de proponer un diseño de muestreo que minimice estos efectos y su posible sesgo.

Las cuatro localidades estudiadas fueron diferentes en las características físicas y vegetales del hábitat. En general, todas tienen una topografía accidentada, se presenta una estación seca muy marcada con baja disponibilidad de agua para los animales, tienen un alto grado de perturbación debido a la deforestación y tala de especies de uso local, hay pastoreo extensivo muy intenso, y presentan un alto grado de marginación social. En particular, el análisis de componentes principales sugiere que las cuatro localidades difieren debido a diferencias en la altitud, en la cobertura térmica que provee la copa de los árboles, y en la cobertura de protección creada por el sotobosque. Las comunidades más disímiles fueron Jolalpan y Huachinantla. Mientras que Mitepec y El Salado presentan condiciones similares entre sí, pero intermedias respecto a las otras dos localidades. Estas diferencias generales y particulares podrían explicar las diferencias en la densidad de venados. Por ejemplo, Mitepec y El Salado el sotobosque está más desarrollado y el grado de perturbación del hábitat es relativamente menor la densidad similar entre sí y fueron mayores respecto a las otras dos localidades. Otro factor que podría afectar a los venados es la accesibilidad a las distintas localidades. En este sentido, coincide que El Salado y Mitepec son las poblaciones más aisladas y donde se presentó la mayor densidad de venados.

Si bien los resultados del presente trabajo son preliminares ya que solo se consideró un año de estudio y se detectaron problemas de tipo metodológico que podrían estar sesgando las estimaciones, la densidad en las cuatro localidades de estudio son bajas. Los principales factores asociados a esta baja densidad podrían ser: 1) alta presión por los pobladores debido a la cacería furtiva, 2) ampliación de la frontera agrícola, y 3) posible competencia entre el ganado y el venado debido a la práctica de libre pastoreo. Esto sugiere establecer políticas de conservación para las poblaciones de venado cola blanca por parte de las instancias gubernamentales correspondientes, así como de los productores y organizaciones no gubernamentales. Se debe hacer un esfuerzo enorme de vigilancia más estricta durante la temporada de caza, así como capacitación a las comunidades para que conformen grupos de vigilancia participativa, y que sean 
apoyados por las autoridades municipales o estatales con el fin de mermar la cacería ilegal. Es urgente además considerar que se conozca la legislación ambiental a través de cursos de educación ambiental hacia los pobladores, así como dar la información necesaria de las áreas en las que se pueden establecer tasas de aprovechamiento fundamentadas con estudios sobre el monitoreo de las poblaciones de venado, estableciendo áreas de veda que permitan el incremento de la población, para que a mediano y/o largo plazo se pueda aprovechar a el venado, así como conservarlo.

\section{AGRADECIMIENTOS}

Agradecemos los comentarios y sugerencias de M. Á. Briones, L. G. Herrera, A. González-Romero, J. Martínez, R. M. González, J. H. García, A. Trujillo y L. Riboni al trabajo de tesis de la primera autora de donde se deriva el presente manuscrito. Varios estudiantes entre los que destacan R. Casco y L. Chong colaboraron durante el trabajo de campo. Agradecemos a los diversos integrantes y autoridades de las comunidades las facilidades para realizar este estudio. El estudio fue financiado parcialmente por la Escuela de Biología de la Benemérita Universidad Autónoma de Puebla, y el Departamento de Biodiversidad y Ecología Animal del Instituto de Ecología A. C.

\section{LITERATURA CITADA}

Castillo, M. A. L. 1998. Estimación poblacional del venado cola blanca en tres localidades de Colima. Tesis de Licenciatura, Benemérita Universidad Autónoma de Puebla, Pue., México.

Centro Nacional de Estudios Municipales. 2000. Los Municipios de Puebla. Colección Enciclopedia de los Municipios de México. Secretaria de Gobernación, México. 1178 pp.

Delfin, C. 2002. Clasificación y evaluación del hábitat como primera fase para el establecimiento y operación de una UMA con fines de aprovechamiento del venado cola blanca mexicano en la Mixteca Poblana. Tesis de Maestría (Manejo de Fauna Silvestre). Instituto de Ecología, A.C. Xalapa, Veracruz, México.

Dietrich, U., J. Francois \& J.C. Moreno. 1990. El conteo de heces fecales ("pellet-groupcount") como método para estimar la densidad poblacional de ungulados: una discusión a base de nuevos datos. In VIII Simposio sobre Fauna Silvestre en México. UNAM y AZARM. México.

Durán, R., A. Hernández \& G. Escalante. 2004. Reproducción y manejo del venado cola blanca (Odocoileus virginianus) en la UAP Amecameca. In: IX Simposio de Venados en México. FMVZ- UNAM. ANGADI. UAEH, Pachuca, Hgo.

Eberhardt, L. \& R. Van Etten. 1956. Evaluation of the pellet group count as a deer census method. Journal of Wildlife Management 20: 70-74.

Ezcurra, E. \& S. Gallina, 1981. Biology and population dynamics of white-tailed deer in Northwestern Mexico. Pp. 78-108. In: Deer Biology, Habitat Requirements, and Management in Western North America (Ffolliott P. F. y S. Gallina, eds.). Instituto de Ecología. México. 125 pp.

Freddy, D. J. \& D. C. Bowden. 1983. Sampling mule deer pellet-group densities in juniperpinyon woodland. Journal of Wildlife Management 47: 476-485. 
Galindo-Leal, C. \& M. Weber. 1998. El Venado de la Sierra Madre Occidental. Ecología, Manejo y Conservación. EDICUSA-CONABIO. México. 272 pp.

Gallina S. A. 1990. El Venado Cola Blanca y su Hábitat en la Michilía, Durango. Tesis de Doctorado, Facultad de Ciencias, UNAM. México. Pp 98.

Gobierno del Estado de Puebla. 1993. Programa de Desarrollo Regional de la Mixteca y la Sierra Negra de Puebla 1993-1999. 56 pp.Griffin, B. y A. Youtie. 1988. Two devices for estimating foliage density and deer hiding cover. Wildlife Society Bulletin 16: 206-211.

INEGI. 1987. Síntesis Geográfica y Nomenclator del Estado de Puebla. INEGI Estado de Puebla. 56 pp.

-1996. Sintesis Geográfica del Estado de Puebla. INEGI, México.

1997. Carta Topográfica 1:250000. Cuernavaca E14-5. México.

1998. Carta Topográfica 1:50000. Temalac E14B81, Tilzapotla E14A79, Huehuetlán E14B71. México.

López-Benítez, J. C., M. C. López-Téllez \& G. Yánez. 2004. Densidad poblacional del venado cola blanca (Odocoileus virginianus mexicanus) en la UMA Tlalhuayan, Chiautla de Tapia, Puebla. In: IX Simposio de Venados en México. FMVZ- UNAM. ANGADI. UAEH, Pachuca, Hgo.

Mandujano, S y S. Gallina. 1995. Comparison of deer censusing methods in a tropical dry forest. Wildlife Society Bulletin 23:180-186.

Martínez-Romero, L. E. 2004. Determinación de fechas de aprovechamiento del venado cola blanca (Odocoileus virginianus) a través de hormonas sexuales y comportamiento. Master Tesis de Maestría (Manejo de Fauna Silvestre). Instituto de Ecología, A. C. Xalapa, Veracruz, México.

Muller-Dombois, D. \& H. Ellenberg. 1974. Aims and Methods of Vegetation Ecology. John Wiley and Sons, N. Y. 547 pp.

Neff, D. J. 1968. The pellet-group count technique for big game trend, census, and distribution: a review. Journal Wildlife Management 32: 597-614.

Ortiz-Martínez, T. J., S. Gallina, M. Briones-Salas \& G. González. 2005. Densidad poblacional y caracterización del hábitat del venado cola blanca (Odocoileus virginianus oaxacensis, Goldman y Kellog, 1940) en un bosque templado de la sierra norte de Oaxaca, México. Acta Zoológica Mexicana (n.s.) 21: 65-78.

Pérez-Mejía, S., S. Mandujano \& L. E. Martínez-Romero. 2004. Tasa de defecación del venado cola blanca, Odocoileus virginianus mexicanus, en cautividad en Puebla. Acta Zoológica Mexicana (n.s.) 20: 167-170.

Rogers, L. L. 1987. Seasonal changes in defecation rates of free-ranging white-tailed deer. Journal of Wildlife Management 51: 330-333.

Romero, S. 1999. Determinación del ciclo reproductivo y ciclo de astas del venado cola blanca (Odocoileus virginianus) en el Parque Estatal General Lázaro Cárdenas, Flor del Bosque, Puebla. Tesis de Licenciatura, Benemérita Universidad Autónoma de Puebla, Pue.

Romero, S. \& L. Martínez-Romero. 1998. Programa de rescate del venado cola blanca (Odocoileus virginianus) en el Parque Estatal Gral. Lázaro Cárdenas Flor del Bosque, en Puebla. VI Simposio sobre Venados en México. UNAM, Xalapa, Ver. 
Romero, S. \&y O. Villarreal-Espino. 2000. Determinación del ciclo reproductivo y ciclo de astas del venado cola blanca (Odocoileus virginianus) en el Parque Estatal General Lázaro Cárdenas, Flor del Bosque, Puebla. VII Simposio sobre Venados en México. UNAM, México, D. F.

Rzedowski, J. 1978. Vegetación de México. Limusa, México, D.F. 432 pp.

Sawyer, T. G., R. L. Marchinton \& W. M. LentZ. 1990. Defecation rates of female whitetailed deer in Georgia. Wildlife Society Bulletin 18: 16-18.

Smith, R. 1968. A comparison of several sizes of circular plots for estimating deer pelletgroups density. Journal of Wildlife Management 32: 585-591.

Valenzuela, D. 1991. Estimación de la densidad y distribución de la población de venado cola blanca (Odocoileus virginianus, Rafinesque 1832) en el bosque la Primavera, Jalisco. Tesis de Licenciatura, Universidad Autónoma de Guadalajara. Guadalajara, Jal., México. pp 83.

Van Etten, R. C. \& C. L. Bennett. 1965. Some sources of error in using pellet-group counts for censusign deer. Journal of Wildlife Management 29:723-729.

Villarreal, J. 1999. Venado Cola Blanca: Manejo y Aprovechamiento Cinegético. Unión Ganadera Regional de Nuevo León. Monterrey, N. L., México. 401pp.

Villarreal-Espino, O. 1998. De Puente de Dios a Casa de Piedra: clasificación tradicional de las astas del venado cola blanca mexicano (Odocoileus virginianus mexicanus), en la región de la Mixteca poblana. VI Simposio sobre Venados en México. UNAM, Xalapa, Ver.

2000. El aprovechamiento sustentable del venado cola blanca mexicano (Odocoileus virginianus mexicanus): una alternativa para el uso del suelo en la región de la Mixteca poblana. VII Simposio sobre Venados en México. UNAM, México, D. F.

2002. El Grand-slam de venado cola blanca mexicano, una alternativa sostenible. Archivos Zootécnicos 51: 187-193.

Villarreal-Espino, O.\& R. V. Guevara. 2002. Distribución regional del venado cola blanca mexicano (Odocoileus virginianus mexicanus) en la Mixteca Poblana, México. Revista de Producción Animal 14: 36-40.

Villarreal-Espino, O. \& M. Marín-Fuentes. 2005. Agua de origen vegetal para el venado cola blanca mexicano. Archivos Zootécnicos 54: 191-196.

Villarreal-Espino, O., J. Villarreal \& P. González. 2000. Primer torneo de canastas de venado cola blanca mexicano (Odocoileus virginianus mexicanus) en Puebla. VII Simposio sobre Venados en México. UNAM, México, D. F.

Wallmo, O. C., A. W. Jackson, T. L. Hailey \& R. L. Carlisle. 1962. Influence of rain on the count of deer pellet groups. Journal of Wildlife Management 26:50-55.

Wigley, T. B. \& M. K. Johnson. 1981. Disappearance rates for deer pellets in the southeast. Journal of Wildlife Management 45:251-253.

Zavala, G. G. 1992. Estimación poblacional del venado cola blanca (Odocoileus virginianus) en la Estación Científica Las Joyas, Reserva de la Biosfera Sierra de Manantlán, Jalisco. Tesis de Licenciatura, Universidad Autónoma de Guadalajara, Jalisco.

Recibido: 2 de julio de 2006

Aceptado: 17 de mayo 2007 